\title{
Helical tomotherapy for single and multiple liver tumours
}

\author{
Tsair-Fwu Lee ${ }^{*+1,2}$, Pei-Ju Chao ${ }^{+1,2}$, Fu-Min Fang², Te-Jen Su1', Stephen W Leung ${ }^{3}$ and Hsuan-Chih Hsu*2
}

\begin{abstract}
Purpose: Dosimetric evaluations of single and multiple liver tumours performed using intensity-modulated helical tomotherapy (HT) were quantitatively investigated. Step-and-shoot intensity-modulated radiotherapy (SaS-IMRT) was used as a benchmark.

Methods: Sixteen patients separated into two groups with primary hepatocellular carcinomas or metastatic liver tumours previously treated using SaS-IMRT were examined and re-planned by HT. The dosimetric indices used included the conformity index (Cl) and homogeneity index (HI) for the planned target volume (PTV), max/mean dose, quality index (Q/), normal tissue complication probability (NTCP), $V_{30 \text { Gy }}$ and $V_{50 \%}$ for the specified organs at risk (OARs). The monitor units per fraction (MU/fr) and delivery time were also analysed.

Results: For the single tumour group, both planning systems satisfied the required PTV prescription, but no statistical significance was shown by the indexes checking. A shorter delivery time and lower MU/fr value were achieved by the SaS-IMRT. For the group of multiple tumours, the average improvement in $\mathrm{Cl}$ and $\mathrm{HI}$ was $14 \%$ and $4 \%$ for HT versus SaSIMRT, respectively. Lower $V_{50 \%}, V_{30}$ Gy and Q/ values were found, indicating a significant dosimetric gain in HT. The NTCP value of the normal liver was $20.27 \pm 13.29 \%$ for SaS-IMRT and $2.38 \pm 2.25 \%$ for $H T$, indicating fewer tissue complications following $\mathrm{HT}$. The latter also required a shorter delivery time.

Conclusions: Our study suggests dosimetric benefits of HT over SaS-IMRT plans in the case of multiple liver tumours, especially with regards sparing of OARs. No significant dosimetric difference was revealed in the case of single liver tumour, but SaS-IMRT showed better efficiency in terms of MU/fr and delivery time.
\end{abstract}

\section{Background}

During the past 20 years, primary liver cancer has ranked the fifth most common malignancy worldwide, the third leading cause of death from malignant neoplasm in Japan in men and the fifth in women $[1,2]$, and the second leading cause of cancer death in Taiwan with a mortality of more than 7,000 cases each year [3]. Several modalities have been used for the treatment of hepatocellular carcinomas (HCC) and metastatic liver tumours [4-10] including surgery, transcatheter arterial chemoembolization (TACE), percutaneous ethanol injection therapy, microwave coagulation therapy, radiotherapy and liver trans-

* Correspondence: tflee@cc.kuas.edu.tw, hsuan5@adm.cgmh.org.tw

${ }^{1}$ Medical Physics and Informatics Lab. (EE), National Kaohsiung University of Applied Sciences, Kaohsiung, Taiwan

2 Chang Gung Memorial Hospital-Kaohsiung Medical Centre, Chang Gung University College of Medicine, Kaohsiung, Taiwan

+ Contributed equally

Full list of author information is available at the end of the article plantation. The role of radiotherapy has been limited because of the poor tolerance of the whole liver to radiation $[11,12]$. With advances in intensity-modulated radiation therapy (IMRT), several reports have indicated increased safety and more promising results in patients with unresectable intrahepatic malignancies treated with radiotherapy to a portion of the liver [6,13-18]. IMRT constitutes an advanced form of the conformal technique and uses inverse planning algorithms and iterative computer-driven optimization to generate treatment fields with varying beam intensity. It has the ability to produce custom-tailored conformal dose distributions around the tumour, although most studies have examined large tumours [19]. IMRT can also be delivered using linac or Hi-Art Helical Tomotherapy (HT) (TomoTherapy, Madison, WI, USA), which creates a more uniform target dose and improves critical organ sparing [16,20-23] with a greater number of degrees of freedom. 
Compared with conventional and other IMRT techniques, HT can potentially produce superior dose distributions (i.e., more uniform dose to the target and lower doses to normal tissues) and is thus being reconsidered for promotion $[21,22,24]$. In this study, we investigated the potential improvement of HT over step-and-shoot (SaS)-IMRT for the treatment of single or multiple liver tumours. HT plans were compared with IMRT plans for sixteen patients previously treated using SaS-IMRT delivery. The HT plans were designed to emulate as closely as possible the goals and constraints used for SaS-IMRT plans. Dose distributions in the planned target volume (PTV) and organs at risk (OARs) were compared according to the isodose distribution and dose-volume histogram (DVH)-based method using several dosimetric parameters including the conformity index $(C I)$ and homogeneity index $(H I)$ for the PTV, max/mean dose, quality index $(Q I)$ for the organs at risk (OARs) [25-29], $V_{30 G y}, V_{50 \%}$, EUD (equivalent uniform dose), and NTCP (normal tissue complication probabilities) for the normal and whole liver. The delivery time and monitor units per fraction (MU/fr) of the two techniques were also compared. SaS-IMRT was used as a benchmark.

\section{Methods}

Study population

Sixteen consecutive patients (six females, ten males) with primary hepatocellular carcinomas (HCC) or metastatic liver tumours previously treated using SaS-IMRT between March 2006 and March 2008 were examined. The patient characteristics and tumour descriptions are presented in Table 1 . The median age was 68 years (range 50-85). Patients were retrospectively grouped to evaluate the influence of the treatment plans. Two groups were formed according to whether they had single (group 1) or multiple (group 2) tumour sites, and interestingly, there were eight in each group. The distributions of clinical stages according to the American Joint Committee on Cancer (AJCC $6^{\text {th }}$ edition) staging system was as follows; I: 1 (6.25\%), II: 3 (18.75\%), III: 5 (31.25\%) and metastasis liver tumour: 7 (43.75\%). Six (37.5\%) were treated with a combination of chemotherapy.

All patients were immobilized using a tailor-made vacuum lock in the supine position with their arms placed on their forehead. The patients were scanned using a CT (Siemens Biograph LSO PET/CT, PA, USA) with a 3-mm slice thickness, containing $512 \times 512$ pixels in each slice. The field of view had a mean dimension of $48 \mathrm{~cm}$.

Treatment plans were originally calculated with the ADAC Pinnacle ${ }^{3}$, version 7.4 (ADAC Inc, CA, USA) treatment-planning system (TPS) on a dose grid of $0.4 \times$ $0.4 \times 0.3 \mathrm{~cm}^{3}$ without DMPO (direct machine parameter optimization). The 5-field and range $4 \times 6 \mathrm{SaS}$-IMRT technique was used with the dose goal for PTV coverage;
Table 1: Patient characteristics $(n=16)$

\begin{tabular}{ll}
\hline Characteristics & No. of patients \\
\hline $\begin{array}{l}\text { Age, median years (range) } \\
\text { Gender }\end{array}$ & $68(50-85)$ \\
$\quad$ Male & $10(62.5 \%)$ \\
Female & $6(37.5 \%)$ \\
Primary HCC (AJCC, 6th edition) & \\
I & $1(6.25 \%)$ \\
II & $3(18.75 \%)$ \\
III & $5(31.25 \%)$ \\
Metastasis liver tumour & $7(43.75 \%)$ \\
Structures (cm 3 ) Mean \pm SD & \\
(range) & \\
PTV & $222.77 \pm 170.35(57.75-726.32)$ \\
Normal liver & $1299.88 \pm 279.03(751.03-1776.16)$ \\
Rt kidney & $132.7 \pm 50.19(35.39-238.91)$ \\
Lt kidney & $147.62 \pm 42.82(78.54-233.17)$ \\
Spinal cord & $14.10 \pm 5.52(4.93-26.44)$ \\
Patient's tumour number & \\
Single (group 1) & $8(50 \%)$ \\
Multiple (group 2) & $8(50 \%)$ \\
\hline Abbrevation:HCC:Hepatocelular & \\
\hline
\end{tabular}

Abbreviation: $\mathrm{HCC}$ : Hepatocellular Carcinoma; $\mathrm{AJCC}=$ American Joint Committee on Cancer; PTV: Planned target volume; Rt: Right side; Lt: Left side;

initial gantry angles of $20^{\circ}, 310^{\circ}, 270^{\circ}, 220^{\circ}$ and $180^{\circ}$ were set. The plan was delivered on an Elekta Precise ${ }^{\text {tw }}$ Linac equipped with an 80-leaf 1-cm MLC in SaS-IMRT mode. Basically, the IMRT planning system tried to achieve the dose goal target coverage while keeping within the dose constraints of OARs by sequential iteration.

\section{PTV and normal organ contouring}

The planned target volume (PTV) structures were created from the gross tumour volume (GTV) structures. Respiratory motion is the main determinant of PTV expansion. PTVs were based on a $5 \mathrm{~mm}$ radial expansion and a $10 \mathrm{~mm}$ craniocaudal expansion. Because respiratory motion has been shown to be greater in the craniocaudal dimension than in the anteroposterior and mediolateral dimensions, an asymmetric expansion was used for the PTV [30-33]. The PTV ranged from 57.75 to 726.32 cc $(222.77 \pm 170.35)$. For dosimetric analysis, the normal liver volume did not include the PTV. The OARs used in this study were as follows: 1) spinal cord-maximum dose $\leq 45$ Gy; 2) kidneys (L \& R)-mean dose to bilateral kidneys must be $<16$ Gy. If only one kidney is present, not more than $15 \%$ of the volume of that kidney can receive $\geq 18$ Gy and no more than $30 \%$ can receive $\geq$ $14 \mathrm{~Gy}$; 3) liver-mean liver dose must be $\leq 25 \mathrm{~Gy}$; 4 ) gas- 
trointestinal system (GIS) (including stomach and small bowels)-maximum dose $\leq 54 \mathrm{~Gy}$; $<10 \%$ of each organ volume can receive between 50 and $53.99 \mathrm{~Gy},<15 \%$ of the volume of each organ can receive between 45 and 49.99 Gy.

\section{Treatment plans}

In the re-planned HT, three main parameters were selected: the field width $(1,2.5$ or $5 \mathrm{~cm})$, pitch (range 0.01-20), and modulation factor (range 1-10). A 2.5-cm field width, a pitch of $0.287(0.86 / 3)$ and a modulation factor of 2 were used in all of the HT plans in this study $[34,35]$. The software version used for this re-planning study was Hi-Art TomoPlan 2.1 (Tomotherapy Inc., Wisconsin, USA). The selection of these three parameter values was based on preliminary planning exercises that showed them to provide a good balance between ability at dose sculpting and treatment efficiency, in terms of treatment duration and feasibility for routine use. In general, small field dimensions, small pitch and large modulation factors mean longer irradiation times and a better ability for the delivery system to sculpt complex dose distributions with steeper dose gradients [16,21,23,24,36]. For all patients, dose calculation was done on the fine grid, which has a resolution of $1.875 \times 1.875 \mathrm{~mm}^{2}$ by the slice thickness of $3 \mathrm{~mm}$ for the dose calculation window of 48 $\times 48 \mathrm{~cm}^{2}(256 \times 256$ pixels $)$. Both planning systems perform iterations during the optimization process. The 0.1 Gy dose bin-size of the dose-volume histograms (DVHs) used in both systems was the same for the subsequent computation of various indices. Plans were run with the goal of delivering the prescribed doses of $60 \mathrm{~Gy} / 30 \mathrm{frac}-$ tions while meeting the normal tissue constraints for conventional treatment. The PTV doses were prescribed to cover over $95 \%$ of the PTV with no greater than a $107 \%$ maximum point dose. Having achieved these objectives, the dose plans were made by the same physicist and approved by the same oncologist, who was specialized in liver tumours. The monitor units per fraction (MU/fr), segments and delivery time taken by the two plans were compared. The patient set-up time was not included.

\section{Plan evaluation}

The HT plans were compared with the SaS-IMRT plans using the following dosimetric parameters:

1. CI: a ratio used to evaluate the goodness of fit of the PTV to the prescription isodose volume in the treatment plans: $C I=V_{P T V} \times \frac{V_{T V}}{T V_{P V}^{2}}$ where $V_{T V}$ is the treatment volume of the prescribed isodose lines; $V_{P T V}$ is the volume of the PTV; and $T V_{P V}$ is the volume of $V_{P T V}$ within the $V_{T V}$. The smaller and closer the value of $C I$ is to 1 , the better the dose conformity [26,37].
2. HI: a ratio used to evaluate the homogeneity of the PTV. $H I=\frac{D_{5 \%}}{D_{95 \%}}$ where $D_{5 \%}$ and $D_{95}$ are the minimum doses delivered to $5 \%$ and $95 \%$ of the PTV. A larger $H I$ indicates poorer homogeneity $[38,39]$.

3. $Q I$ : an index used to evaluate the difference in the maximum or mean absorbed dose at serial or parallel OARs, respectively, between HT and SaS-IMRT plans $[22,40]$.

$$
Q I_{\text {Serial }}=\frac{D_{\max }^{h t}}{D_{\max }^{i m r t}}, \quad Q I_{\text {Parallel }}=\frac{D_{\text {mean }}^{h t}}{D_{\text {mean }}^{\text {imrt }}} .
$$

4. $V_{50 \%}$ : the percentage volume receiving a dose greater than or equal to $50 \%$ of the prescribed dose for a normal liver.

5. $V_{30}$ Gy: the percentage volume receiving a dose greater than or equal to $30 \mathrm{~Gy}$ for the whole liver.

6. EUD: equivalent uniform dose, the original definition of the EUD was derived on the basis of a mechanistic formulation using a linear-quadratic cell survival model [41]. Subsequently, Niemierko and Emami suggested a phenomenological model of the form [42]:

$$
E U D=\left(\sum_{i=1}\left(v_{i} D_{i}^{a}\right)\right)^{1 / a}
$$

where $\alpha$ is a unitless model parameter that is specific to the normal structure or tumour of interest, and $v_{i}$ is unitless and represents the $i$ th partial volume receiving dose $D_{i}$ in Gy. Since the relative volume of the whole structure of interest corresponds to 1 , the sum of all partial volumes $v_{i}$ will equal 1 . For normal tissues, the EUD represents the uniform dose that leads to the same probability of injury as the examined inhomogeneous dose distribution.

7. NTCP: an EUD-based normal tissue complication probability (NTCP) was used. Niemierko proposed parameterization of the dose-response characteristics using the logistic function $[42,43]$ :

$$
N T C P=\frac{1}{1+\left(\frac{T D_{50}}{E U D}\right)^{4 \gamma 50}}
$$

where $T D_{50}$ is the tolerance dose for a $50 \%$ complication rate at a specific time interval (e.g., 5 years in the Emami et al. normal tissue tolerance data [44]) when the whole organ of interest is homogeneously irradiated, and $\gamma_{50}$ is a unitless model parameter that is specific to the normal 
structure and describes the slope of the dose-response curve. Niemierko and Emami suggested that the parameters of $\alpha$ and $\gamma_{50}$ should be used in the EUD-based NTCP model. The values of $\alpha, \gamma_{50}$, and $T D_{50}$ used in this study were 3, 3, and 40 Gy respectively, and were based on the Emami data, calculating the BED as 2 Gy/fraction with an $\alpha / \beta$ ratio of $2[42,44]$. The Matlab-2009a software (MathWorks, Inc., Natick, Massachusetts) was used for EUDbased NTCP and CERR (computational environment for radiotherapy research) calculations [45].

\section{Statistical analyses}

The mean values (standard deviation) of the dosimetric data for the sixteen patients were analysed using the paired Wilcoxon signed-rank test to compare the difference between HT and SaS-IMRT. A two-tailed value of $p$ $<0.05$ was deemed to indicate statistical significance. The SPSS-15.0 software was used for data processing (SPSS, Inc., Chicago, IL).

\section{Results \\ PTV analysis}

The isodose distributions in the axial plane and the DVHs of the PTV and OARs for one typical case in each group plan using both systems are shown in Figs. 1 and 2.

Table 2 gives the dose statistics for the PTV for each group with HT and SaS-IMRT.

For group 1, the mean $\mathrm{V}_{95 \%}$ and $\mathrm{V}_{100 \%}$ for the desired PTV coverage was $99.44 \pm 0.72$ and $97.26 \pm 1.13$ in the HT plans, and $99.63 \pm 0.51$ and $97.84 \pm 0.99$ in the SaSIMRT plans, respectively, with no significant differences between plans. For the hot spot checking, the mean $\mathrm{V}_{107 \%}$ for the desired PTV was $0.00 \pm 0.00$ with HT and $8.75 \pm$ 4.94 with SaS-IMRT respectively, indicating significantly better homogeneity of the PTV with HT $(\mathrm{p}<0.05)$. ( $\mathrm{V}_{\mathrm{x} \%}$ : volume receiving $\geq \mathrm{x} \%$ of the prescribed dose).

The mean CI for group 1 was $1.21 \pm 0.07$ with HT and $1.30 \pm 0.05$ with SaS-IMRT, indicating a significantly better conformity of the PTV with HT $(\mathrm{p}<0.05)$ The average improvement in CI was 7\% for HT. The mean HI was 1.04 \pm 0.01 for HT and $1.06 \pm 0.01$ for SaS-IMRT; this difference was statistically significant $(p<0.05)$ with a $2 \%$ improvement in HT.

For group 2, the mean $\mathrm{V}_{95 \%}$ and $\mathrm{V}_{100 \%}$ for the desired PTV coverage was $99.09 \pm 0.45$ and $96.20 \pm 0.70$ in the HT plans, and $98.47 \pm 0.69$ and $96.13 \pm 1.10$ in the SaSIMRT plans, respectively, with no significant difference between plans. For the hot spot checking, the mean $\mathrm{V}_{107 \%}$ for the desired PTV was $0.30 \pm 0.58$ with $\mathrm{HT}$ and $16.62 \pm$ 2.38 with SaS-IMRT respectively, indicating significantly better homogeneity of the PTV with HT ( $<<0.05)$.

The mean $C I$ was $1.25 \pm 0.11$ with $\mathrm{HT}$ and $1.43 \pm 0.07$ with SaS-IMRT, indicating significantly better conformity of the PTV with HT ( $<<0.05)$. The average improvement in $C I$ was $14 \%$ for $H$ T versus SaS-IMRT. The mean $H I$ for group 2 was $1.06 \pm 0.01$ for HT and $1.10 \pm 0.02$ for SaSIMRT; this difference was statistically significant $(p<$ 0.05 ) with a $4 \%$ improvement in HT.

\section{Dosimetry of OARs}

The dose statistics of the specified OARs are summarized in Table 3. For group 1, the mean dose, $V_{50 \%}$ and NTCP value of the normal liver did not differ significantly between the HT and SaS-IMRT plans $(p>0.05)$. Similarly there was no significant difference between plans in the $V_{30 \text { Gy }}$ value of the whole liver $(p>0.05)$ or the max/mean dose of the other four OARs (R/Lt kidneys, GIS, and spinal cord) $(p>0.05)$.

For group 2, the mean dose, $V_{50 \%}$ and NTCP value of the normal liver were significantly lower in the HT plans versus the SaS-IMRT plans $(p<0.05)$. The $V_{50 \%}$ value of the normal liver was $36.46 \pm 4.92 \%$ for HT and $51.74 \pm$ $11.46 \%$ for SaS-IMRT, indicating an approximate reduction of $15 \%$ in HT. With regards tissue complications the NTCP value of the normal liver was $2.38 \pm 2.25 \%$ for HT and $20.27 \pm 13.29 \%$ for SaS-IMRT, indicating an approximate reduction of $18 \%$ in $\mathrm{HT}$ (NTCP for liver failure). The $V_{30 \text { Gy }}$ value of the whole liver differed significantly between plans $(p<0.05)$. The mean value of $V_{30}$ Gy for the whole liver was $43.91 \pm 10.43 \%$ for $\mathrm{HT}$ and $55.00 \pm$ $14.28 \%$ for SaS-IMRT, indicating an approximate $11 \%$ reduction in HT. The $\max /$ mean dose of the following three OARs (R/Lt kidneys and GIS) did not differ significantly. The maximum dose of the spinal cord was $18.08 \pm$ 5.38 for HT and $23.55 \pm 8.65$ Gy for SaS-IMRT. These results indicate a significant dosimetric gain in $\mathrm{HT}$ and a reduced dose to sensitive structures.

\section{QI analysis for the OARs}

The QI values of the OARs for group 1 and group 2 are listed in Table 4; the kidneys were excluded in the QI calculation as the test results did not differ significantly.

For group 1, of the two serial OARs, the spinal cord showed the most notable improvement $[Q I=0.86 \pm 0.47]$ followed by GIS $[Q I=0.91 \pm 0.23]$, indicating an approximate $14 \%$ reduction in maximal dose in the spinal cord and a $9 \%$ reduction in the GIS in the HT versus SaSIMRT plans, respectively $(p>0.05)$. Of the only parallel organ (normal liver) calculated, the $Q I_{\text {Parellel }}$ was $0.95 \pm$ 0.20 , indicating an approximate mean dose reduction of $5 \%$ in the normal liver in the HT versus SaS-IMRT plans.

For group 2, of the two serial OARs, the spinal cord showed the most notable improvement $[Q I=0.83 \pm 0.30]$ followed by the GIS [QI $=0.95 \pm 0.12]$, indicating an approximate $17 \%$ reduction in maximal dose in the spinal cord and a $5 \%$ reduction in the GIS in the HT versus SaS- 


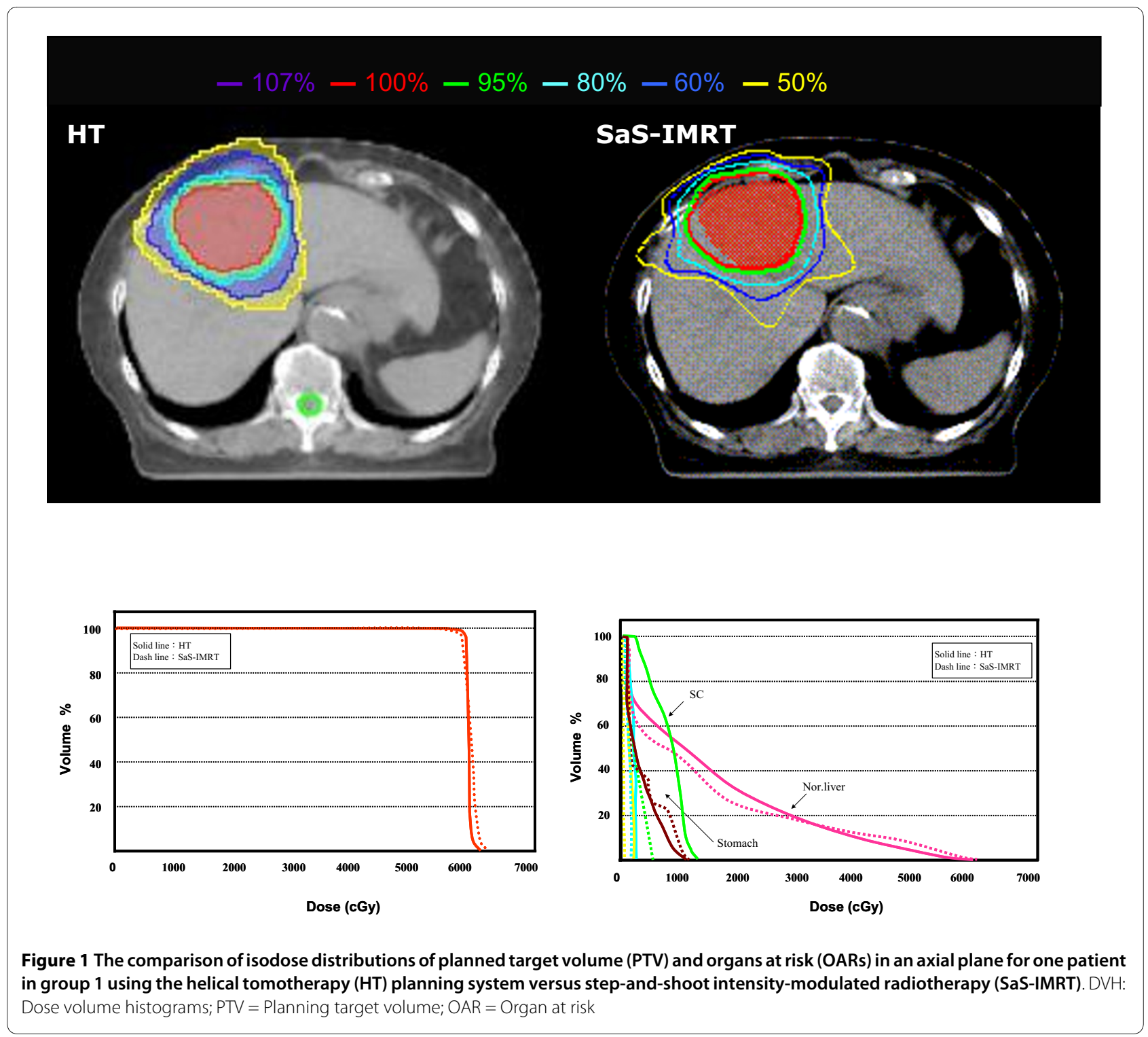

IMRT plans, respectively. Of the only parallel organ (normal liver) calculated, the $Q I_{\text {Parellel }}$ was $0.93 \pm 0.17$, indicating an approximate mean dose reduction of $7 \%$ in the normal liver by HT.

For the whole study cohort, of the two serial OARs, the spinal cord showed the most notable improvement [QI= $0.85 \pm 0.38]$ followed by the GIS [QI $=0.93 \pm 0.18]$, indicating an approximate $15 \%$ reduction in maximal dose in the spinal cord and a 7\% reduction in the GIS by HT. Of the only parallel (normal liver) organ calculated, the $Q I$ was $0.93 \pm 0.17$, indicating an approximate mean dose reduction of $7 \%$ in the normal liver by $\mathrm{HT}$.

\section{$\mathrm{MU} / \mathrm{fr}$ and delivery time}

The MU/fr and delivery time of the sixteen patients with HT versus SaS-IMRT are compared in Table 5. For group 1 , the mean delivery time was $4.4 \pm 1.4$ min (range 2.9-
6.3) for HT and $3.3 \pm 1.4 \mathrm{~min}$ (range 1.9-5.2) for SaSIMRT, with a significant difference between these values $(p=0.00)$. The mean MU/fr used was $5135 \pm 1678$ for $\mathrm{HT}$, which was significantly higher than the mean $\mathrm{MU} / \mathrm{fr}$ of $343 \pm 120$ in SaS-IMRT $(p<0.05)$.

For group 2, the mean delivery time was $4.7 \pm 0.8 \mathrm{~min}$ (range 3.3-5.7) for HT and 6.2 $\pm 1.4 \mathrm{~min}$ (range 4.8-8.8) for SaS-IMRT. A significant difference was observed between these values $(p<0.05)$. The mean MU/fr used was $5529 \pm 960$ for HT, which was significantly higher than the mean MUs of $461 \pm 242$ in SaS-IMRT $(p<0.05)$.

\section{Discussion}

The benefits of improved dose homogeneity and better sparing of critical organs in HT compared with conventional linac-based IMRT have been reported in prostate 

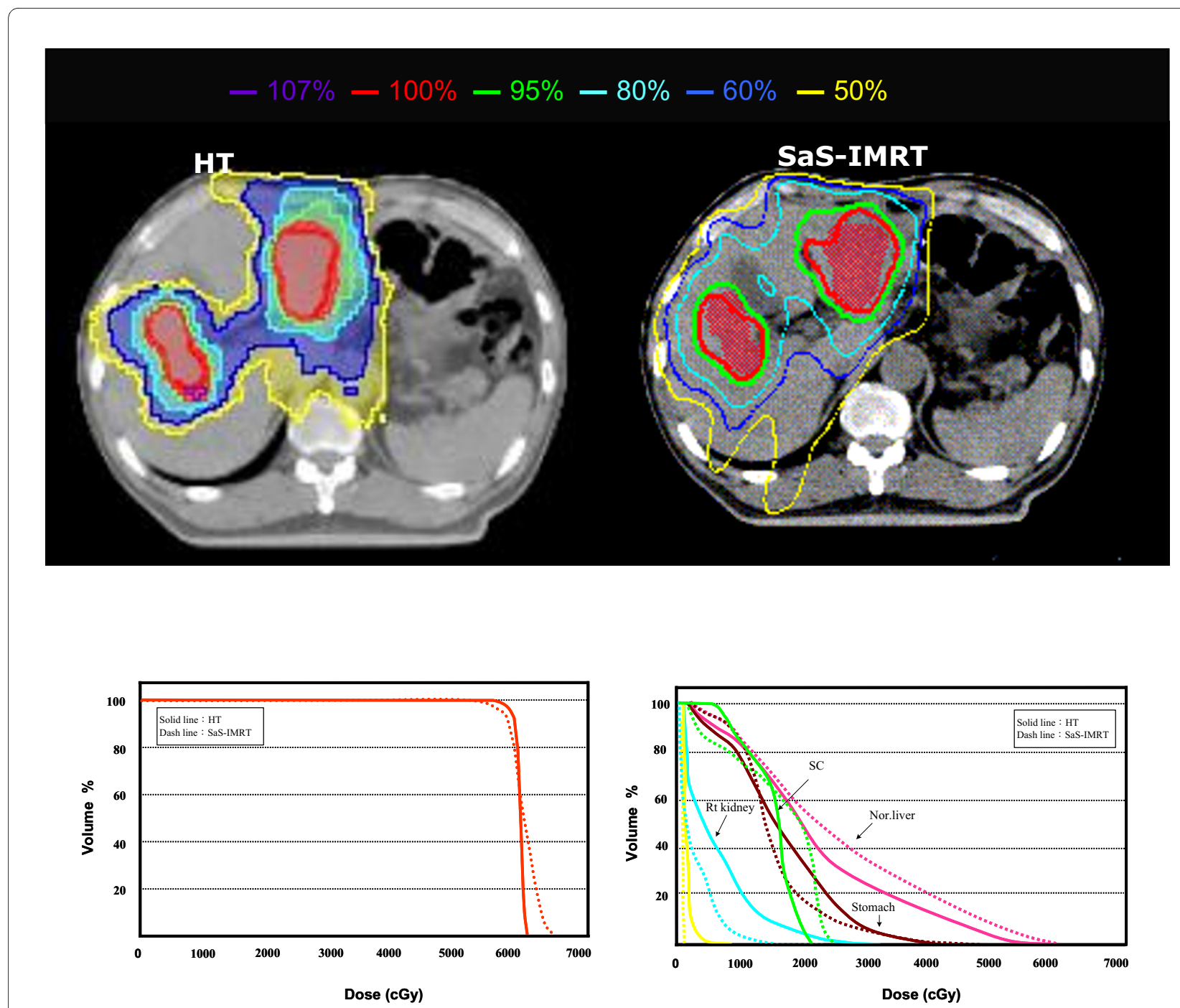

Figure 2 The comparison of isodose distributions of planned target volume (PTV) and organs at risk (OARs) in an axial plane for one patient in group 2 using the helical tomotherapy (HT) planning system versus step-and-shoot intensity-modulated radiotherapy (SaS-IMRT). DVH: Dose volume histograms; PTV = Planning target volume; OAR = Organ at risk

cancer [46], intracranial tumours [24], nasopharyngeal carcinoma [22] and other head and neck cancers [47,48], and breast cancer [13]. However, these benefits of IMRT and HT are generally achieved at the cost of a greater volume of normal tissue in the irradiated volume receiving a low dose $[29,49]$. In addition, radiotherapy for liver tumours is largely limited by the dose to the surrounding normal tissues, primarily the residual normal liver tissue.

One of the major objectives of this study was to determine the achievable gain of HT in single and multiple liver tumour irradiations against a well-investigated and routinely-used clinical technique, SaS IMRT, delivered in a conventional way with SaS-IMRT planning and an Elekta Precise delivery system. Sixteen cases in two groups were investigated in this study. The HT plans had a slightly significantly better conformity and homogeneity to the PTV than SaS-IMRT plans in the whole cohort. However, the dosimetric advantages of the two plans were inconsistent for individual OARs and other indices.

We demonstrated that HT plans significantly improved the conformity index (improvement ratio: 7 and 14\%) and homogeneity index (improvement ratio: 2 and 4\%) of the PTV compared with SaS-IMRT plans in group 1 and 2, respectively.

However, the difference between the mean/maximal doses of OARs was not statistically significant in group 1, indicating no difference in OARs sparing. Sparing was found in the normal liver with mean values of $Q I-1=0.95$ \pm 0.20 and $Q I-2=0.90 \pm 0.14$, and in the spinal cord with 
Table 2: The dosimetric results of PTV between HT and SaS-IMRT plans for two groups

\begin{tabular}{|c|c|c|c|c|}
\hline Parameter & objective & HT & SaS-IMRT & $p$ \\
\hline \multicolumn{5}{|c|}{ Group 1-single tumour group } \\
\hline $\mathrm{V}_{95 \%}$ & 100 & $99.44 \pm 0.72(97.97-100.00)$ & $99.63 \pm 0.51(98.78-100.00)$ & $\mathrm{n} / \mathrm{a}$ \\
\hline$V_{100 \%}$ & 95 & $97.26 \pm 1.13(95.87-98.79)$ & $97.84 \pm 0.99(96.50-99.07)$ & $\mathrm{n} / \mathrm{a}$ \\
\hline $\mathrm{V}_{107 \%}$ & 0 & $0.00 \pm 0.00$ & $8.75 \pm 4.94(2.30-15.14)$ & $<0.05$ \\
\hline $\mathrm{Cl}$ & 1 & $1.21 \pm 0.07(1.15-1.37)$ & $1.30 \pm 0.05(1.23-1.40)$ & $<0.05$ \\
\hline $\mathrm{HI}$ & 1 & $1.04 \pm 0.01(1.02-1.05)$ & $1.06 \pm 0.01(1.02-1.07)$ & $<0.05$ \\
\hline \multicolumn{5}{|c|}{ Group 2-multiple tumours group } \\
\hline $\mathrm{V}_{95 \%}$ & 100 & $99.09 \pm 0.45(98.43-99.95)$ & $98.47 \pm 0.69(97.35-99.25)$ & $\mathrm{n} / \mathrm{a}$ \\
\hline$V_{100 \%}$ & 95 & $96.20 \pm 0.70(95.60-97.86)$ & $96.13 \pm 1.10(94.80-97.77)$ & $\mathrm{n} / \mathrm{a}$ \\
\hline $\mathrm{V}_{107 \%}$ & 0 & $0.30 \pm 0.58(0.00-1.60)$ & $16.62 \pm 2.38(13.70-20.53)$ & $<0.05$ \\
\hline $\mathrm{Cl}$ & 1 & $1.25 \pm 0.11(1.15-1.47)$ & $1.43 \pm 0.07(1.37-1.57)$ & $<0.05$ \\
\hline $\mathrm{HI}$ & 1 & $1.06 \pm 0.01(1.02-1.07)$ & $1.10 \pm 0.02(1.08-1.12)$ & $<0.05$ \\
\hline
\end{tabular}

Abbreviation: SaS-IMRT: Step-and-shoot intensity-modulated radiotherapy; $\mathrm{HT}$ : Helical tomotherapy; $\mathrm{V}_{\mathrm{x} \%}$ : volume receiving $\geq \mathrm{x} \%$ of the prescribed dose; $\mathrm{Cl}$ : Conformity index; $\mathrm{HI}$ : Homogeneity index; $n / a$ : not statistical significance; statistical significance $(p<0.05)$ is reported between couples from the paired Wilcoxon signed-rank test analysis.

mean values of $Q I-1=0.86 \pm 0.47$ and $Q I-2=0.83 \pm 0.30$ in group 2, indicating a dosimetric gain in the $\mathrm{HT}$ plans.

In $V_{30 \text { Gy }}$ and $V_{50 \%}$ analysis, HT showed a significant dosimetric gain in group 2 . The results showed that a better (lower) dose was received in HT than that in group 1; for group 2, the mean value of $V_{50 \%}$ of the whole liver was $36.46 \pm 4.92$ for HT and 51.74 \pm 11.46 for SaS-IMRT, indicating an approximate reduction of $15.3 \%$ in HT. The mean value of $V_{30 \mathrm{~Gy}}$ of the normal liver was $43.91 \pm 10.43$ for $\mathrm{HT}$ and $55.00 \pm 14.28$ for SaS-IMRT, indicating an approximate reduction of $11.1 \%$ in $\mathrm{HT}$. These results showed a significant dosimetric gain in $\mathrm{HT}$ and a reduced mean liver dose.

In clinical practice, the $\mathrm{V}_{50 \%}$ (fraction of normal liver treated to at least $50 \%$ of the isocentre dose) and the $V_{30}$ Gy (the percentage volume receiving a dose greater than or equal to $30 \mathrm{~Gy}$ for the whole liver) are the most commonly used indicators for the dose given. According to the Yonsei University guidelines [50], if the percentage of normal liver volume receiving $50 \%$ of the isocentre dose was less than $25 \%$, the total dose was increased to 59.4 Gy; if the percentage was $25 \%$ to $49 \%$, the dose was 45 to $54 \mathrm{~Gy}$; if the percentage was $50 \%$ to $75 \%$, the dose was 30.6 to $45 \mathrm{~Gy}$, and if the dose was more than $75 \%$, no treatment was administered. They showed that the parameter $\mathrm{V}_{50 \%}$ can be divided into four categories and used to predict acceptable liver toxicity. In group 2, the $\mathrm{V}_{50 \%}$ value of normal liver was $36.46 \pm 4.92 \%$ for $\mathrm{HT}$ and $51.74 \pm 11.46 \%$ for SaS-IMRT, indicating an opportunity for dose escalation by HT versus SaS-IMRT plans. The NTCP value of the normal liver was $2.38 \pm 2.25 \%$ for HT and $20.27 \pm 13.29 \%$ for SaS-IMRT, indicating that a reduction in tissue complications may be achieved by HT versus SaS-IMRT plans.

Kim et al. demonstrated that the $\mathrm{V}_{30 \text { Gy }}$ appears to be a useful dose-volumetric parameter for predicting the risk of radiation-induced hepatic toxicity (RIHT). In their report, grade 2 or worse RIHT was observed in only 2 out of 85 patients $(2.4 \%)$ with a whole liver volume receiving $30 \mathrm{~Gy}\left(\mathrm{~V}_{30 \mathrm{~Gy}}\right.$, whole liver) of $\leq 60 \%$, and in 11 out of 20 patients $(55.0 \%)$ with greater than $60 \%(p<0.001)$ [12].

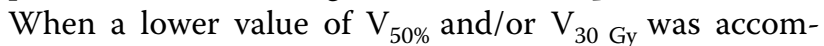
plished, a higher PTV dose could be given. As a result, a lower $\mathrm{V}_{50 \%}$ and/or $\mathrm{V}_{30 \text { Gy }}$ can be achieved with HT for the treatment of multiple liver tumours than with SaS-IMRT. Consequently, a higher dose can be given and a higher response can be achieved when HT is selected.

The overall delivery time and average MU/fr used in the HT plans were significantly higher than for SaSIMRT plans, which are consistent with the results of several studies [13,22,24,47-49]. The delivery time depended on the limitations of gantry rotation and dose prescription in the HT system, while a speed limitation on gantry rotation exists in the HT system. An interesting result occurred in this study in that a contrary result was found in group 2 due to the geometry of the multiple site distribution. The mean delivery time in group 2 was $4.7 \pm 0.8$ min (range 3.3-5.7) for HT and 6.2 \pm 1.4 min (range 4.88.8) for SaS-IMRT. This difference was significant ( $p=0$. 01).

We also found that both planning systems satisfied the required PTV prescription, but that better dose conformity and homogeneity were achieved with the HT com- 
Table 3: Dosimetric statistics for the specified OARs

\begin{tabular}{|c|c|c|c|c|}
\hline Structure & Parameter & HT & SaS-IMRT & $\mathbf{p}$ \\
\hline \multicolumn{5}{|c|}{ Group 1-single tumour group } \\
\hline \multirow[t]{4}{*}{ Normal liver } & Mean (Gy) & $18.24 \pm 6.73(10.84-31.09)$ & $20.01 \pm 7.86(8.37-31.20)$ & $\mathrm{n} / \mathrm{a}$ \\
\hline & $V_{50 \%}(\%)$ & $19.17 \pm 5.62(10.83-22.50)$ & $22.19 \pm 7.13(14.17-31.25)$ & $\mathrm{n} / \mathrm{a}$ \\
\hline & EUD & $23.68 \pm 5.14(16.60-33.97)$ & $29.11 \pm 5.46(21.52-37.14)$ & $<0.05$ \\
\hline & NTCP & $1.69 \pm 4.30(0.003-12.33)$ & $6.80 \pm 9.86(0.06-29.07)$ & 0.051 \\
\hline Whole liver & $\mathrm{V}_{30 \mathrm{~Gy}}(\%)$ & $36.41 \pm 14.88(16.45-62.00)$ & $39.44 \pm 16.57(16.94-62.06)$ & $\mathrm{n} / \mathrm{a}$ \\
\hline Lt kidney & Mean (Gy) & $2.48 \pm 2.43(0.30-6.44)$ & $2.83 \pm 3.61(0.17-9.00)$ & $\mathrm{n} / \mathrm{a}$ \\
\hline Rt kidney & Mean (Gy) & $4.13 \pm 3.09(0.42-8.03)$ & $5.55 \pm 4.55(0.15-10.57)$ & $\mathrm{n} / \mathrm{a}$ \\
\hline GIS & $\operatorname{Max}(G y)$ & $30.18 \pm 18.17(8.65-52.56)$ & $32.67 \pm 17.27(11.77-53.45)$ & $\mathrm{n} / \mathrm{a}$ \\
\hline Spinal cord & Max (Gy) & $15.30 \pm 9.14(5.12-34.28)$ & $22.05 \pm 11.10(4.58-34.78)$ & $\mathrm{n} / \mathrm{a}$ \\
\hline \multicolumn{5}{|c|}{ Group 2-multiple tumours group } \\
\hline \multirow[t]{4}{*}{ Normal liver } & Mean (Gy) & $25.89 \pm 3.43(18.89-28.45)$ & $29.73 \pm 6.71(15.54-36.96)$ & $<0.05$ \\
\hline & $\mathrm{V}_{50 \%}(\%)$ & $36.46 \pm 4.92(29.17-41.67)$ & $51.74 \pm 11.46(37.5-69.17)$ & $<0.05$ \\
\hline & EUD & $28.09 \pm 3.23(21.95-31.87)$ & $34.68 \pm 3.80(27.77-38.43)$ & $<0.05$ \\
\hline & NTCP & $2.38 \pm 2.25(0.07-6.15)$ & $20.27 \pm 13.29(1.23-38.33)$ & $<0.05$ \\
\hline Whole liver & $\mathrm{V}_{30 \mathrm{~Gy}}(\%)$ & $43.91 \pm 10.43(23.12-53.42)$ & $55.00 \pm 14.28(27.11-74.97)$ & $<0.05$ \\
\hline Lt kidney & Mean (Gy) & $4.18 \pm 2.94(0.66-9.21)$ & $2.60 \pm 2.03(0.37-6.97)$ & $\mathrm{n} / \mathrm{a}$ \\
\hline Rt kidney & Mean (Gy) & $6.11 \pm 4.16(0.99-12.38)$ & $6.45 \pm 4.76(0.93-14.58)$ & $\mathrm{n} / \mathrm{a}$ \\
\hline GIS & $\operatorname{Max}(G y)$ & $39.59 \pm 12.42(21.42-53.20)$ & $42.05 \pm 12.36(19.67-52.78)$ & $\mathrm{n} / \mathrm{a}$ \\
\hline Spinal cord & $\operatorname{Max}(G y)$ & $18.08 \pm 5.38(10.58-28.19)$ & $23.66 \pm 8.65(8.96-32.20)$ & $<0.05$ \\
\hline
\end{tabular}

pared to SaS-IMRT plans in the two groups. No significant was shown for OARs sparing in group 1, especially if the tumour is leaning against the body surface. As the result, general SaS-IMRT can meet the prescription requirements like the $\mathrm{HT}$ did, but shown more efficiency in $\mathrm{MU} / \mathrm{fr}$ used and delivery time saved than HT in group 1.

We did not aim to perform a strict comparison of the two systems, but to retrospectively evaluate the dosimet- ric difference for the 16 patients that had been successfully treated with step-and-shoot IMRT and re-planned in a routinely-used helical tomotherapy based upon the same planning CT scan; the dose plans were made by the same physicist and approved by the same oncologist who was specialized in liver tumours. We paid careful attention to reducing biases in this study. However, there are some limitations with regard to our results, and although we used the same resolution, voxel size, and binning of

Table 4: The dosimetric comparisons of QI between HT and SaS-IMRT plans

\begin{tabular}{|c|c|c|c|}
\hline Variables of OARs & QI-1 & QI-2 & QI-whole cohort \\
\hline \multicolumn{4}{|l|}{ QI of parallel organ } \\
\hline Normal Liver & $0.95 \pm 0.20(0.61-1.30)$ & $0.90 \pm 0.14(0.76-1.21)$ & $0.93 \pm 0.17(0.61-1.30)$ \\
\hline \multicolumn{4}{|l|}{ Ql of serial organ } \\
\hline SC & $0.86 \pm 0.47(0.16-1.45)$ & $0.83 \pm 0.30(0.56-1.52)$ & $0.85 \pm 0.38(0.16-1.52)$ \\
\hline GIS & $0.91 \pm 0.23(0.64-1.36)$ & $0.95 \pm 0.12(0.75-1.11)$ & $0.93 \pm 0.18(0.64-1.36)$ \\
\hline
\end{tabular}

Abbreviation: HT: Helical tomotherapy; SaS-IMRT: Step-and-shoot intensity-modulated radiotherapy; Ql: Quality index; QI-1: QI-single tumour group; QI-2: QI-multiple tumours group; SC: Spinal cord; GIS: Gastrointestinal system (including stomach and small bowels); 
Table 5: Delivery time and MU/fr used in HT and SaS-IMRT plans

\begin{tabular}{|c|c|c|c|c|c|c|c|c|}
\hline & \multicolumn{4}{|c|}{ Group 1-single tumour group } & \multicolumn{4}{|c|}{ Group 2-multiple tumours group } \\
\hline & \multicolumn{2}{|c|}{ HT } & \multicolumn{2}{|c|}{ SaS-IMRT } & \multicolumn{2}{|c|}{ HT } & \multicolumn{2}{|c|}{ SaS-IMRT } \\
\hline & DT (min) & MU/fr & DT (min) & $\mathbf{M U} / \mathbf{f r}$ & DT (min) & $\mathbf{M U} / \mathbf{f r}$ & DT (min) & MU/fr \\
\hline (range) & $(2.9-6.3)$ & $(3461-7404)$ & $(1.9-5.2)$ & $(227-577)$ & $(3.3-5.7)$ & $(3904-6704)$ & $(4.8-8.8)$ & $(148-698)$ \\
\hline
\end{tabular}

Abbreviation: HT: Helical tomotherapy; SaS-IMRT: Step-and-shoot intensity-modulated radiotherapy; DT: delivery time; MU/fr: Monitor units used per fraction; Patient setup time was not included.

the DVHs in both systems, and the same software (CERR), an intrinsic difference in the calculation algorithms or TPS optimization modules (such as DMPO) might produce different results.

\section{Conclusions}

Our study suggests the dosimetric benefits of HT over SaS-IMRT plans in the group with multiple liver tumours, especially with regards sparing of OARs, as it significantly reduced the $\mathrm{V}_{50 \%}$ and $\mathrm{V}_{30 \text { Gy }}$ to the normal liver and whole liver respectively. In addition a reduction in the NTCP value indicates that fewer tissue complications may arise in HT plans. Although there was no significant difference in the group with single liver tumour, IMRT showed better efficiency in terms of the MU/fr and delivery time used.

\section{Competing interests}

The authors declare that they have no competing interests.

\section{Authors' contributions}

TFL and PJC: idea and concept. FMF; TJS and SWL: design and development of study. PJC and HCH: statistical analysis. TFL: writing of manuscript and study coordinator. FMF and $\mathrm{HCH}$ : final revision of manuscript. All authors read and approved the final manuscript.

\section{Acknowledgements}

The authors thank the anonymous reviewers for their helpful comments on the original manuscript and Dr. YJ Huang, Ms. HM Ting and Mr. MH Liu for their technical support and data collection. This study was supported financially, in part, by grants from the CGMH (CMRPG890061) and NSC (98-2221-E-151-038).

\section{Author Details}

${ }^{1}$ Medical Physics and Informatics Lab. (EE), National Kaohsiung University of Applied Sciences, Kaohsiung, Taiwan, ${ }^{2}$ Chang Gung Memorial HospitalKaohsiung Medical Centre, Chang Gung University College of Medicine, Kaohsiung, Taiwan and ${ }^{3} Y u a n ' s$ General Hospital, Kaohsiung, Taiwan

Received: 26 April 2010 Accepted: 24 June 2010

Published: 24 June 2010

\section{References}

1. Kiyosawa K, Umemura T, Ichijo T, Matsumoto A, Yoshizawa K, Gad A, Tanaka E: Hepatocellular carcinoma: Recent trends in Japan. Gastroenterology 2004, 127:S17-S26.

2. Thomas MB, Abbruzzese JL: Opportunities for targeted therapies in hepatocellular carcinoma. Journal of Clinical Oncology 2005, 23:8093.
3. Kao Y-H, Chen C-N, Chiang J-K, Chen S-S, Huang W-W: Predicting Factors in the Last Week of Survival in Elderly Patients with Terminal Cancer: A Prospective Study in Southern Taiwan. Journal of the Formosan Medical Association 2009, 108:231-239.

4. Cha CH, Ruo L, Fong Y, Jarnagin WR, Shia J, Blumgart LH, DeMatteo RP: Resection of hepatocellular carcinoma in patients otherwise eligible for transplantation. Ann Surg 2003, 238:315.

5. Dae Yong K, Won P, Do Hoon L, Joon Hyoek L, Byung Chul Y, Seung Woon P, Kwang Cheol K, Tae Hyun K, Yong Chan A, Seung Jae H: Threedimensional conformal radiotherapy for portal vein thrombosis of hepatocellular carcinoma. Cancer 2005, 103:2419-2426.

6. Fuss M, Salter BJ, Herman TS, Thomas JCR: External beam radiation therapy for hepatocellular carcinoma: Potential of intensity-modulated and image-guided radiation therapy. Gastroenterol 2004, 127:S206-S217.

7. Tse RV, Guha C, Dawson LA: Conformal radiotherapy for hepatocellular carcinoma. Crit Rev Oncol Hematol 2008, 67:113-123.

8. Wang X, Krishnan S, Zhang X, Dong L, Briere T, Crane CH, Martel M, Gillin M, Mohan R, Beddar S: Proton radiotherapy for liver tumors: dosimetric advantages over photon plans. Med Dosim 2008, 33:259-267.

9. Ohto M, Yoshikawa M, Saisho H, Ebara M, Sugiura N: Nonsurgical treatment of hepatocellular carcinoma in cirrhotic patients. World $J$ Surg 1995, 19:42-46.

10. Tsuzuki T, Sugioka A, Ueda M, lida S, Kanai T, Yoshii H, Nakayasu K: Hepatic resection for hepatocellular carcinoma. Surgery 1990, 107:511.

11. Cha CH, Saif MW, Yamane BH, Weber SM: Hepatocellular carcinoma: current management. Curr Prob/ Surg 47:10-67.

12. Kim TH, Kim DY, Park J-W, Kim SH, Choi J-I, Kim HB, Lee WJ, Park SJ, Hong EK, Kim C-M: Dose-volumetric parameters predicting radiation-induced hepatic toxicity in unresectable hepatocellular carcinoma patients treated with three-dimensional conformal radiotherapy. Int J Radiat Oncol Biol Phys 2007, 67:225-231.

13. Caudrelier JM, Morgan SC, Montgomery L, Lacelle M, Nyiri B, MacPherson M: Helical tomotherapy for locoregional irradiation including the internal mammary chain in left-sided breast cancer: Dosimetric evaluation. Radiother Oncol 2009, 90:99-105.

14. Cheng JC-H, Wu J-K, Huang C-M, Liu H-S, Huang DY, Tsai SY, Cheng SH, Jian JJ-M, Huang AT: Dosimetric analysis and comparison of threedimensional conformal radiotherapy and intensity-modulated radiation therapy for patients with hepatocellular carcinoma and radiation-induced liver disease. Int J Radiat Oncol Biol Phys 2003, 56:229-234.

15. Esiashvili N, Koshy M, Landry J: Intensity-modulated radiation therapy. Curr Probl Cancer 28:47-84.

16. Mavroidis P, Ferreira BC, Shi C, Lind BK, Papanikolaou N: Treatment plan comparison between helical tomotherapy and MLC-based IMRT using radiobiological measures. Phys Med Biol 2007, 52:3817-3836.

17. Dawson LA, Normolle D, Balter JM, McGinn CJ, Lawrence TS, Ten Haken RK: Analysis of radiation induced liver disease using the Lyman NTCP model* 1. Int J Radiat Oncol Biol Phys 2002, 53:810-821.

18. Dawson LA, Ten Haken RK, Lawrence TS: Partial irradiation of the liver. Elsevier 2001:240-246. 
19. Kang M, Kim M, Kim S, Choi J: High dose radiotherapy for massive hepatocellular carcinoma (HCC) with intensity-modulated radiation therapy (IMRT). Int J Radiat Oncol Biol Phys 2009, 75:S281-S281.

20. Jang JW, Kay CS, Bae SH, Choi JY, Jung HJ, You CR, Kim CW, Cho SH, Yoon SK, Han JY, et al.: 378 Simultaneous multitarget irradiation using helical tomotherapy for advanced hepatocellular carcinoma with multiple extrahepatic metastases. Journal of Hepatology 2008, 48:S147-S147.

21. Lawrence JA, Forrest LJ: Intensity-modulated radiation therapy and helical tomotherapy: Its origin benefits, and potential applications in veterinary medicine. Vet Clin Small Anim 2007, 37:1151-1165.

22. Lee TF, Fang FM, Chao PJ, Su TJ, Wang LK, Leung SW: Dosimetric comparisons of helical tomotherapy and step-and-shoot intensitymodulated radiotherapy in nasopharyngeal carcinoma. Radiother Oncol 2008, 89:89-96.

23. Mackie TR: History of tomotherapy. Phys Med Biol 2006, 51:R427-453.

24. Han C, Liu A, Schultheiss TE, Pezner RD, Chen YJ, Wong JYC: Dosimetric comparisons of helical tomotherapy treatment plans and step-andshoot intensity-modulated radiosurgery treatment plans in intracranial stereotactic radiosurgery. Int J Radiat Oncol Biol Phys 2006, 65:608-616

25. Baltas D, Kolotas C, Geramani K, Mould RF, loannidis G, Kekchidi M, Zamboglou N: A conformal index (COIN) to evaluate implant quality and dose specification in brachytherapy. Int I Radiat Oncol Biol Phys 1998, 40:515-524

26. Leung LHT, Kan MWK, Cheng ACK, Wong WKH, Yau CC: A new dosevolume-based plan quality index for IMRT plan comparison. Radiother Oncol 2007, 85:407-417.

27. Paddick I: A simple scoring ratio to index the conformity of radiosurgical treatment plans. J Neurosurg 2000, 93:219-222.

28. Riet Av, Mak ACA, Moerland MA, Elders LH, van der Zee W: A conformation number to quantify the degree of conformality in brachytherapy and external beam irradiation: Application to the prostate. Int J Radiat Oncol Biol Phys 1997, 37:731-736.

29. Yang R, Xu S, Jiang W, Xie C, Wang J: Integral dose in three-dimensional conformal radiotherapy, intensity-modulated radiotherapy and helical tomotherapy. Clini Oncol 2009, 21:706-712.

30. Wagman R, Yorke E, Ford E, Giraud P, Mageras G, Minsky B, Rosenzweig K Respiratory gating for liver tumors: use in dose escalation. Int J Radiat Oncol Biol Phys 2003, 55:659-668

31. Baisden JM, Reish AG, Sheng K, Larner JM, Kavanagh BD, Read PW: Dose as a function of liver volume and planning target volume in helical tomotherapy, intensity-modulated radiation therapy-based stereotactic body radiation therapy for hepatic metastasis. Int J Radiat Oncol Biol Phys 2006, 66:620-625.

32. Clifford M, Banovac F, Levy E, Cleary K: Assessment of hepatic motion secondary to respiration for computer assisted interventions. Computer Aided Surgery 2002, 7:291-299.

33. Nakashige A, Horiguchi J, Tamura A, Asahara T, Shimamoto F, Ito K: Quantitative measurement of hepatic portal perfusion by multidetector row $\mathrm{CT}$ with compensation for respiratory misregistration. Bri J Radiol 2004, 77:728.

34. Sterzing F, Sroka-Perez G, Schubert K, Münter MW, Thieke C, Huber P, Debus Jg, Herfarth KK: Evaluating target coverage and normal tissue sparing in the adjuvant radiotherapy of malignant pleural mesothelioma: Helical tomotherapy compared with step-and-shoot IMRT. Radiotherapy and Oncology 2008, 86:251-257.

35. Kissick MW, Fenwick J, James JA, Jeraj R, Kapatoes JM, Keller H, Mackie TR, Olivera G, Soisson ET: The helical tomotherapy thread effect. Medical Physics 2005, 32:1414-1423.

36. Balog J, Holmes T, Vaden R: A helical tomotherapy dynamic quality assurance. Med Phys 2006, 33:3939-3950.

37. Penagaricano J, Yan Y, Shi C, Linskey M, Ratanatharathorn V: Dosimetric comparison of helical tomotherapy and gamma knife stereotactic radiosurgery for single brain metastasis. Radiat Oncol 2006, 1:26.

38. Ling C, Archambault Y, Bocanek J, Zhang P, LoSasso T, Tang G: Scylla and charybdis: longer beam-on time or lesser conformality-the dilemma of tomotherapy. Int J Radiat Oncol Biol Phys 2009, 75:8-9.

39. Rahimian J, Chen J, Rao A, Girvigian M, Miller M, Greathouse H: Geometrical accuracy of the Novalis stereotactic radiosurgery system for trigeminal neuralgia. J Neurosurg 2004, 101:351-355.

40. Lee TF, Chao PJ, Wang CY, Lan JH, Huang YJ, Hsu HC, Sung CC, Su TJ, Lian $S L$, Fang FM: Dosimetric comparison of helical tomotherapy and dynamic conformal arc therapy in stereotactic radiosurgery for vestibular schwannomas. Medical Dosimetry . doi:10.1016/ j.meddos.2009.11.005

41. Wu Q, Mohan R, Niemierko A, Schmidt-Ullrich R: Optimization of intensity-modulated radiotherapy plans based on the equivalent uniform dose* 1 . International Journal of Radiation Oncology* Biology* Physics 2002, 52:224-235.

42. Gay HA, Niemierko A: A free program for calculating EUD-based NTCP and TCP in external beam radiotherapy. Physica Medica 2007, 23:115-125

43. Niemierko A: A generalized concept of equivalent uniform dose (EUD). Med Phys 1999, 26:1100.

44. Emami B, Lyman J, Brown A, Coia L, Goitein M, Munzenrider JE, Shank B, Solin $\perp$, Wesson M: Tolerance of normal tissue to therapeutic irradiation. International Journal of Radiation Oncology Biology Physics 1991, 21:109.

45. Deasy J, Blanco A, Clark V: CERR: a computational environment for radiotherapy research. Medical Physics 2003, 30:979.

46. Aoyama H, Westerly DC, MacKie TR, Olivera GH, Bentzen SM, Patel RR, Jaradat H, Tome WA, Ritter MA, Mehta MP: Integral radiation dose to normal structures with conformal external beam radiation. Int $J$ Radiat Oncol Biol Phys 2006, 64:962-967.

47. Sheng K, Molloy JA, Read PW: Intensity-modulated radiation therapy (IMRT) dosimetry of the head and neck: A comparison of treatment plans using linear accelerator-based IMRT and helical tomotherapy. Int J Radiat Oncol Biol Phys 2006, 65:917-923.

48. van Vulpen M, Field C, Raaijmakers CPJ, Parliament MB, Terhaard CHJ MacKenzie MA, Scrimger R, Lagendijk JJW, Fallone BG: Comparing stepand-shoot IMRT with dynamic helical tomotherapy IMRT plans for head-and-neck cancer. Int J Radiat Oncol Biol Phys 2005, 62:1535-1539.

49. Purdy JA: Dose to normal tissues outside the radiation therapy patient's treated volume: A review of different radiation therapy techniques. Health Physics 2008, 95:666.

50. Lee IJ, Seong J, Shim SJ, Han KH: Radiotherapeutic parameters predictive of liver complications induced by liver tumor radiotherapy. Int J Radiat Oncol Biol Phys 2009, 73:154-158.

doi: $10.1186 / 1748-717 \mathrm{X}-5-58$

Cite this article as: Lee et al. Helical tomotherapy for single and multiple liver tumours Radiation Oncology 2010, 5:58

\section{Submit your next manuscript to BioMed Central and take full advantage of:}

- Convenient online submission

- Thorough peer review

- No space constraints or color figure charges

- Immediate publication on acceptance

- Inclusion in PubMed, CAS, Scopus and Google Scholar

- Research which is freely available for redistribution 